\title{
Evaluation of Smear Negative Pulmonary Tuberculosis Before and After Antibiotic Use: The Case of Four Public Health Centers of Addis Ababa, Ethiopia
}

\author{
Shemsu Kedir Juhar ${ }^{1}$, Awad Mohammed Amdalla ${ }^{2}$, Negash Nurahmed ${ }^{1}$, Mulusew Getahun ${ }^{1}$, Ahmed \\ Mohammed $^{1}$, Sisay Kebede Gebregeorgis ${ }^{2}$, Yemsirach Reta Silishe², Kassu Destæ2 \\ ${ }^{1}$ Ethiopian Public Health Institute, Ethiopia; ${ }^{2}$ College of Health Sciences Department of Medical Laboratory Sciences, \\ Addis Ababa University, Ethiopia
}

\begin{abstract}
Background: Diagnosis of pulmonary tuberculosis in sputum smear-negative patients is challenging in today's medical practice. As a result, Diagnosis of SNPT mostly supported on clinical and radiographic elements in our country set up.

Methods: A prospective cross-sectional study on the burden of smear-negative pulmonary tuberculosis from a total of 220 suspected pulmonary tuberculosis patients conducted in four public health centers of Addis Ababa, Ethiopia between May 25, 2017, and September 30, 2017. Study participants sputum sample were screened for acid-fast bacilli by direct smear microscopy two times before and after antibiotic administration and demographic data was taken. All smear negative morning sputum samples were further processed for culture using solid Lowenstein-Jensen medium at the St. Peter TB Specialized Hospital TB laboratory. Statistical analysis was done to predict the burden of smearnegative pulmonary tuberculosis.

Results: 206 (93.6\%) patients with suspected pulmonary tuberculosis have had negative smear results for acid-fast bacilli. $16(7.76 \%)$ and $14(6.8 \%)$ patients whose smears were negative for acid-fast bacilli found to be positive for Mycobacterium culture before and after taking broad-spectrum antibiotics respectively. Abnormal chest X-ray findings were observed in 25 (54.3\%) out of 46 patients. Night sweat and weight loss have shown a significant association with culture-confirmed SNPT.

Conclusion: The study showed $92.2 \%$ smear-negative pulmonary tuberculosis cases were still etiologically unexplained by culture. Therefore, there is a need to improve practice on sputum sample collection, processing and using other existing better diagnostic approaches for the diagnosis of SNPT in the Ethiopian setting to avoid a missed diagnosis of patients.
\end{abstract}

Keywords: Mycobacterium tuberculosis; Antibiotics; Smear; Nontuberculosis mycobacterium

\section{INTRODUCTION}

Tuberculosis (TB) is a chronic infectious disease caused by Mycobacterium tuberculosis (MTB). It typically affects the lungs (pulmonary TB) but can affect other parts of the body as well (extrapulmonary TB). The disease is spread via droplet infection when people with pulmonary TB expel the bacilli while coughing, sneezing, talking, etc. Without treatment, mortality rates are high. Treatment using combinations of anti-TB drugs, developed in the 1940s and 1950s, can dramatically reduce mortality rates [1].

For decades, the diagnosis of pulmonary tuberculosis (TB) has relied on the search for Acid-Fast Bacilli (AFB) in sputum

Corresponding author: Shemsu Kedir Juhar, Ethiopian Public Health Institute, Ethiopia, Tel: +251913768756; E-mail: shemsukedir1@gmail.com

Received date: May 16, 2019; Accepted date: July 11, 2019; Published date: July 20, 2019

Citation: Juhar SK, Amdalla AM, Nurahmed N, Getahun M, Mohammed A, Gebregeorgis SK, et al. (2019) Evaluation of Smear Negative Pulmonary Tuberculosis Before and After Antibiotic Use: The Case of Four Public Health Centers of Addis Ababa, Ethiopia. J Trop Dis 7:322.

Copyright: (C) 2019 Juhar SK et al. This is an open-access article distributed under the terms of the Creative Commons Attribution License, which permits unrestricted use, distribution and reproduction in any medium, provided the original author and source are credited. 
smears, a procedure that has a low sensitivity of only $50-60 \%$ (Cultures positive for $M$. tuberculosis provides a definitive diagnosis of TB $[2,3]$.

Ethiopia is one of the 22 high burden countries for tuberculosis. According to the WHO global TB report 2015, there were an estimated 200,000 (207 per 100,000 populations) incident cases of TB in Ethiopia in 2014. There were an estimated 32,000 deaths (33 per 100,000 populations) due to TB excluding HIV related deaths. According to 2011 health and health-related indicators of the $\mathrm{FMOH}$, tuberculosis is the third leading cause of death in Ethiopia (Figure 1) [4].

Several algorithms and clinical scoring systems based on local epidemiology have been developed to predict smear-negative tuberculosis. Few of these have been validated within the local context. However, in areas where smear-negative tuberculosis poses a major public-health problem, these algorithms may be useful to the national tuberculosis program by providing a starting point for developing their own context-specific diagnostic guidelines [2].

In the absence of positive sputum smears for AFB, at the primary care level, most cases of smear-negative pulmonary tuberculosis are diagnosed on the basis of clinical and radiological indicators followed by health care worker decision.

The diagnosis of pulmonary tuberculosis in sputum smearnegative patients is a challenge for today's medical practice. And it has been associated with poor treatment outcomes and excessive mortality, particularly in high Human Immunodeficiency Virus (HIV) prevalent settings, diagnosis mostly supported on clinical and radiographic elements [5].

Low detection of smear-positive and over-diagnosis of smearnegative Pulmonary Tuberculosis (PTB) are major problems in Ethiopia. Non-adherence to diagnostic guidelines could be contributing to the poor detection of tuberculosis [5].

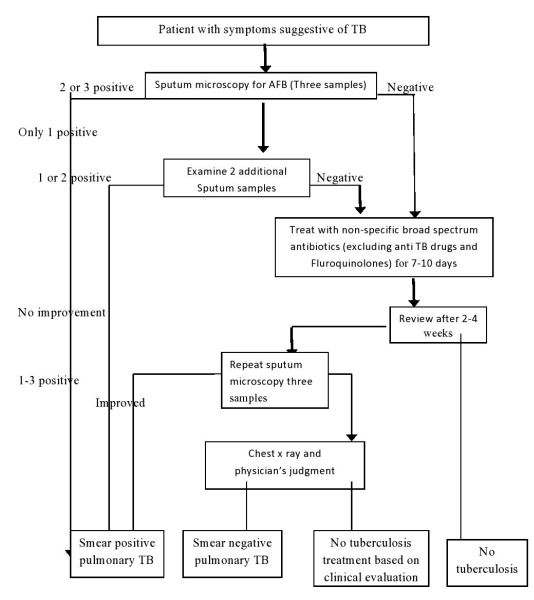

Figure 1: Algorithm and diagnosis of Tb, adopted from Guidelines for clinical and programmatic management of TB, TB/HIV, and leprosy in Ethiopia, FMOH, 2013.

So this study was done to determine the prevalence of sputum smear-negative pulmonary tuberculosis focusing before and after administration of antibiotics and investigate the value of the clinical and radiological parameter for diagnosis of smearnegative pulmonary tuberculosis.

\section{MATERIALS AND METHODS}

A prospective cross-sectional study was conducted in Kazanchis, Hiwet Amba, Kirkos and Feres Meda public health centers in Kirkos sub-city, Addis Ababa, Ethiopia from May 25, 2017, to September 30, 2017. During the study there were seven public health centers within the sub-city, all of them working sputum AFB microscopy and participate in external quality assessment.

AFB microscopy is routinely done for 5, 2, 4, and $3 \mathrm{~TB}$ suspected patients per day on average in Kazanchis, Hiwet Amba, Kirkos and Feres Meda public health centers respectively. There were about 154 registered TB patients taking anti TB drugs in the health centers. Among TB patients 48, 60, and 46 are SPPT, SNPT, and extrapulmonary TB respectively.

Patients visiting the selected public health centers were used as source population and adult patients age above 18 years, male and female, not taking anti TB drug, not on antibiotics trial within a month and suspect of having pulmonary tuberculosis and fulfill the inclusion criteria, who agreed to participate in the study and fill consent form were included and considered as the study subject.

Non-random consecutive sample size of 220 was used for study subjects from selected health centers until the required sample size obtained. Four health centers were selected from Kirkos subcity conveniently

Adult male and female Patients, age greater than 18 years, presenting with cough >or equal to 2 weeks, not on antibiotics within a month, who give three times sputum for AFB (ZN); and who gave a written informed consent to participate in the study were involved as inclusion criteria.

Patients with known tuberculosis, on anti TB for treatment or prophylaxis, have extrapulmonary and confirmed smear-positive pulmonary TB were excluded.

Clinical and socio-demographic data were collected from patients using structured questionnaires through an interview by data collectors for those who are in inclusion criteria and chest $\mathrm{X}$-ray examination and culture results were transferred to the questionnaire.

Then patients were given spot morning spot sputum sample for smear microscopy and only morning sputum was used for LJ culture. Sputum was collected on clean, sterile leak-proof, wide mouth container (falcon tube for morning sputum) and clearly labeled with patient identification information.

First, Consecutive Spot morning spot Sputum specimens were collected from suspect patients of pulmonary tuberculosis, who did not use broad-spectrum antibiotics at least for one month for any disease. AFB microscopy was done and morning sputum was inoculated into LJ solid culture media for those who are AFB smear negative.

Then Sputum samples were collected again from patients after the use of broad-spectrum antibiotics for 7-10 days. AFB microcopies were done for sputum and smear negative morning 
sputum was transported to St. Peter TB Specialized Hospital TB laboratory for culture. Morning Sputum sample was transported using a vaccine carrier with an ice pack of $2^{\circ} \mathrm{C}-8^{\circ} \mathrm{C}$ and double packaging system. Sputum sample that was not inoculated soon were stored in $2^{\circ} \mathrm{C}-8^{\circ} \mathrm{C}$ for not more than 5 days.

All sputum samples received at the selected public health centers (spot morning spot) were smeared, fixed with light and stained with ZN staining technique. Direct sputum microscopic examination was performed for Acid Fast Bacilli (AFB) using light microscopy as the national TB diagnosis guideline. These AFB procedures were done before and after antibiotic use for all study participants.

All morning sputum samples from patients were processed according to standard procedures using NALC-NAOH. Processed Concentration of the sputum samples was inoculated into two slants of Lowenstein-Jensen (LJ) and incubated at $37^{\circ} \mathrm{C}$ with weekly examination for growth. Specimens that did not have colonies growing at eight weeks were defined as negative.

Specimens with any growth were examined by Ziehl-Neelsen (ZN) microscopy and positive isolates were identified by the SD BIOLINE TB Ag MPT64 RAPID an immunochromatographic assay [6].

And drug sensitivity tests were done for culture positive result samples, using a line probe assay of molecular technique for rifampicin and isoniazid.

\section{Safety precautions}

Microscopy smear (ZN) were done using safety practice and use sprite lamp between smear and laboratory personnel. Sputum processing, decontamination, inoculum preparation for culture were performed in a suitable bio-safety level III TB laboratory, BSC 2, dedicated for mycobacterium work with negative air pressure and with an appropriate ventilation system. Access to the room is restricted when work is in progress and proper use of personal protective equipment and disposal of waste while handling specimens were practiced.

\section{Quality control}

Quality control slides of positive and negative were done for AFB staining every week to ensure the quality of the reagents. For culture, confirm the performance characteristics of each lot of LJ media by using well-characterized lab strains of $\mathrm{M}$. tuberculosis complex and one NTM. A random sample of the LJ batch at $37^{\circ} \mathrm{C}$ for 48 hours and evaluate each slant for growth. Data collections practices were checked randomly for data collectors.

\section{Data processing and statistical analysis}

All data were double entered into an excel spreadsheet, cleaned, verified and then transferred for statistical calculation in Stata 15 (state corporation, college station, TX)

Univariate Odds Ratios (OR) with 95\% Confidence Intervals (CI) were calculated to evaluate the possible association between smear-negative PTB and different variables in terms of demographics, clinical symptoms, chest X-ray, and AFB laboratory test results. All variables evaluated in univariate analysis were then included in a multivariate regression model to identify potential predictors for PTB by calculating adjusted ORs with 95\% Confidence Intervals (CI). Chi-square test was used to assess whether the difference between the value obtained is significant. Sensitivity, specificity positive and negative predictive value also calculated. All statistical tests were considered significant if the two-sided $\mathrm{p}$-value is $<0.05$.

\section{Ethical clearance}

The research proposal was evaluated by the Departmental Research and Ethics Review Committee of Department of Medical Laboratory Science, College of health science, Addis Ababa University, St. Peter Specialized Hospital and also by Addis Ababa City Administration Health Bureau.

\section{Dissemination of results}

The findings of the study were communicated to respective health centers and patients for possible intervention. The paper was presented to the Department of Medical Laboratory Science. The findings also disseminated to St. Peter Specialized TB Hospital. The paper will be presented to seminars or workshop to disseminate the findings and published in peerreviewed journals.

\section{RESULTS}

\section{Socio-demographic characteristics for study participants before administration of antibiotics}

A total of 220 pulmonary tuberculosis suspect patients were included in the study. Fourteen individuals excluded due to their AFB smear-positive result. Two hundred six AFB smearnegative pulmonary tuberculosis suspects were included in the study. The mean and median age of the study participant was 42 \pm 16.4 and 40 respectively. About $59.3 \%(122 / 206)$ of the study participants were females. The majority of TB suspects live in urban (Addis Ababa) 93.7\% and 33.5\% (69/206) study participant were governmental workers as shown in (Tables 1 and 2).

Table 1: Smear negative Pulmonary Tuberculosis suspected study participant symptom characteristics before the administration of antibiotics, 2017.

\begin{tabular}{lll}
\hline Variable & $\begin{array}{l}\text { Frequency } \\
\mathrm{N}=206\end{array}$ & Percent (\%) \\
\hline Cough >2 weeks & 199 & 96.6 \\
\hline Presence of sputum & 165 & 80 \\
\hline Night sweat & 70 & 34 \\
\hline Tiredness & 136 & 66 \\
\hline Shortness of breath & 84 & 40.7 \\
\hline Presence of blood in sputum & 12 & 5.8 \\
\hline
\end{tabular}




\begin{tabular}{lcc}
\hline Headache & 144 & 69.9 \\
\hline Weight loss & 45 & 21.8 \\
\hline Fever & 123 & 59.7 \\
\hline
\end{tabular}

Table 2: Socio-demographic characteristics of smear-negative pulmonary tuberculosis suspects among SNPT suspect's patients before administration of antibiotics at selected public health centers at Addis Ababa, Ethiopia, 2017.

\begin{tabular}{lllll}
\hline Variable & $\begin{array}{l}\text { Frequency } \\
\text { total }\end{array}$ & $\begin{array}{l}\text { Percent } \\
(\%)\end{array}$ & $\begin{array}{l}\text { SNPT } \\
\text { (Culture }\end{array}$ & $\begin{array}{l}\text { Culture } \\
\text { negative }\end{array}$ \\
$\mathrm{N}=206$ & & $\begin{array}{l}\text { positive }) \\
\mathrm{n}=190 \text { cases }\end{array}$ & $(92.2 \%)$ \\
& & $(7.8 \%)$ & \\
& & & & \\
& & &
\end{tabular}

Health center

\begin{tabular}{lcccc}
\hline Feres Meda & 61 & 29.6 & $7(3.4)$ & $54(26.2)$ \\
\hline Hiwet Amba & 39 & 19 & $4(1.9)$ & $35(17)$ \\
\hline Kirkos & 54 & 26.2 & $3(1.5)$ & $51(24.8)$ \\
\hline Kazanchis & 52 & 25.2 & $2(1)$ & $50(24.2)$ \\
\hline Sex & & & & \\
\hline Male & 84 & 40.7 & $7(3.4)$ & $77(37.4)$ \\
\hline Female & 122 & 59.3 & $9(4.4)$ & $113(54.8)$ \\
\hline
\end{tabular}

Age group

\begin{tabular}{lllll}
\hline $19-30$ & 38 & 18.4 & $1(0.5)$ & $37(18)$ \\
\hline $31-40$ & 53 & 25.7 & $3(1.5)$ & $50(24.3)$ \\
\hline $41-50$ & 63 & 30.6 & $6(2.9)$ & $57(27.6)$ \\
\hline$>50$ & 52 & 25.3 & $6(2.9)$ & $4622.3)$ \\
\hline
\end{tabular}

Occupation

\begin{tabular}{lllll}
\hline Farmer & 7 & 3.4 & 0 & $7(3.4)$ \\
\hline Merchant & 37 & 18 & $4(1.9)$ & $33(16)$ \\
\hline Governmental & 69 & 33.5 & $9(4.4)$ & $60(29.1)$ \\
\hline Worker other & 93 & 45.1 & $3(1.5)$ & $90(43.7)$ \\
\hline
\end{tabular}

Living resident

\begin{tabular}{lllll}
\hline Urban & 193 & 93.7 & $16(7.8)$ & $177(85.9)$ \\
\hline Rural & 13 & 6.3 & 0 & $13(6.3)$ \\
\hline
\end{tabular}

\section{Prevalence of smear-negative pulmonary tuberculosis before and after the use of antibiotics}

From a total of 242 study participants, 220 individuals finished the study. About 22 study individuals could not come after antibiotic use and exclude from the study. AFB microscopy did for the study participants and smear-negative pulmonary tuberculosis suspects before antibiotic use were $93.6 \%$ (206/220). About 6.4\% (14/220) were smeared positive PTB and were excluded from the study. From AFB smear-negative TB suspect patients, the prevalence of culture-confirmed SNPT was $7.8 \%(16 / 206)$ and $0.48 \%(1 / 206)$ was Nontuberculosis Mycobacterium before TB suspect patients using broad-spectrum antibiotics as shown in Table 3.

Table 3: Culture results for sputum Smear Negative Pulmonary Tuberculosis suspects patients before and after antibiotics use for a study participant, 2017.

\begin{tabular}{lllll}
\hline \multirow{2}{*}{ Culture results } & \multicolumn{2}{c}{ Before antibiotic } & \multicolumn{2}{c}{ After antibiotic } \\
\cline { 2 - 5 } & Frequency & $\%$ & Frequency & $\%$ \\
\hline Positive & & & & \\
\hline $\begin{array}{l}\text { Mycobacterium } \\
\text { tuberculosis }\end{array}$ & 16 & 7.76 & 14 & 6.8 \\
\hline $\begin{array}{l}\text { Non tuberculosis } \\
\text { mycobacterium }\end{array}$ & 1 & 0.48 & - & - \\
\hline $\begin{array}{l}\text { Negative } \\
\text { Contaminated }\end{array}$ & 10 & 86.9 & 180 & 87.8 \\
\hline
\end{tabular}

AFB microscopy was done for 206 study participants after finished using broad-spectrum antibiotics for 7-10 days. One previously AFB negative sputum sample found to be scanty positive, and the rest 205 samples were still negative. Two previously culture-positive samples were one negative and the other contaminated and could not repeat due to the loss of a patient.

Study participant whose AFB smear result positive were put on anti $\mathrm{TB}$ regimen based on Ethiopian National guidelines. Majority of AFB smear-negative study participant were taking Amoxicillin $500 \mathrm{mg} 164$ (79.6\%), erythromycin $500 \mathrm{mg} 21$ (10.2\%), Doxycycline $100 \mathrm{mg}$ 6.8\% (14/206) and 3.4\% (7/206) prescribed another drug like Augmentin $625 \mathrm{mg}$. About 22 study participant was not back after antibiotic use and excluded from the study. 
Line probe assay done for the 14 culture positive samples to test drug sensitivity test after antibiotics use and showed no MDR cases, all showed a susceptible pattern of Rifampicin and Isoniazid line for wild type and no mutation.

\section{Clinical characteristics and chest X-ray results}

Prediction for determination of smear negative culture positive was done Using unadjusted logistic regression model. The binary regression identified that night sweat (OR (95\% $C I)=11.083(3.065-40.08)$, weight loss $(O R \quad(95 \% \mathrm{CI})=15.945$ (4.88-52.067)), fatigue (OR $(95 \% \mathrm{CI})=1.58(1.424-1.77)$ and fever (OR $\quad(95 \% \quad \mathrm{CI})=7.928 \quad(6.125-10.26) \quad$ were independently associated with confirmed SNPT. whereas other symptom had no significant association with culture-confirmed SNPT $(p>0.05)$ as shown in Table 4.

Table 4: Bi-variates regression analysis of clinical characteristics predictive of smear negative culture positive PTB, 2017.

\begin{tabular}{llllll}
\hline Variables & Total N=206 & SNPT n=16 & No SNPT n=190 & OR (95\%CI) & p-value \\
\hline Cough >2 weeks & $199(96.6 \%)$ & $16(100 \%)$ & $183(96.3 \%)$ & $1.038(1.01-1.068)$ & 0.419 \\
\hline Presence of sputum & $165(80 \%)$ & $10(62.5 \%)$ & $155(81.6 \%)$ & $0.417(0.14-1.203)$ & 0.097 \\
\hline Night sweat & $70(34 \%)$ & $13(81.2 \%)$ & $57(81.4 \%)$ & $11.08(3.065-40.08)$ & 0.0001 \\
\hline Fatigue & $136(66 \%)$ & $16(100 \%)$ & $120(63.1)$ & $1.58(1.424-1.77)$ & 0.002 \\
\hline Shortness of breath & $84(40.7 \%)$ & $12(75 \%)$ & $72(37.9 \%)$ & $5.401(1.69-16.2)$ & 0.052 \\
\hline Hemoptysis & $12(5.8 \%)$ & $4(25 \%)$ & $8(4.2 \%)$ & $6.96(1.84-26.2)$ & 0.062 \\
\hline Fever & $123(59.7 \%)$ & $16(100 \%)$ & $107(56.3 \%)$ & $7.92(6.125-10.26)$ & 0.001 \\
\hline Headache & $13(81.25 \%)$ & $144(69.9 \%)$ & $131(68.9 \%)$ & $2.22(0.586-7.65)$ & 0.243 \\
\hline Weight loss & $45(21.8 \%)$ & $12(75 \%)$ & $33(17.3 \%)$ & $15.94(4.88-52.06)$ & 0.001 \\
\hline Abnormal CXR & $14(87.5 \%)$ & $25(54.3 \%)$ & $11(5.8 \%)$ & $3.104(1.71-5.63)$ & 0.48 \\
\hline
\end{tabular}

A multivariate regression model was developed from significant and clinically important covariates. Multivariate regression analysis found that night sweat (OR $(95 \% \quad \mathrm{CI})=0.113$ (0.027-0.474) and weight loss (OR (95\% CI) $=0.72(0.019-0.272)$ have shown significantly associated with confirmed SNPT as shown in Table 5.

Table 5: Multivariate analysis of clinical characteristics predictive of smear negative culture positive PTB, 2017.

\begin{tabular}{llllll}
\hline Variables & $\begin{array}{l}\text { Total } \\
\mathbf{n}=206\end{array}$ & $\begin{array}{l}\text { SNPT } \\
\mathbf{n}=16\end{array}$ & $\begin{array}{l}\text { No } \\
\text { SNPT } \\
\mathbf{n}=190\end{array}$ & OR (95\% CI) & $\begin{array}{l}\text { p- } \\
\text { value }\end{array}$ \\
\hline $\begin{array}{l}\text { Night } \\
\text { sweat }\end{array}$ & $70(34 \%)$ & $\begin{array}{l}13 \\
(81.2 \%)\end{array}$ & $\begin{array}{l}57 \\
(81.4 \%)\end{array}$ & $\begin{array}{l}0.113 \\
(0.027-0.474)\end{array}$ & 0.003 \\
\hline Fatigue & $\begin{array}{l}136 \\
(66 \%)\end{array}$ & $\begin{array}{l}16 \\
(100 \%)\end{array}$ & $\begin{array}{l}120 \\
(63.1)\end{array}$ & $1.11(1.110-1.21)$ & 0.051 \\
\hline $\begin{array}{l}\text { Weight } \\
\text { loss }\end{array}$ & $\begin{array}{l}45 \\
(21.8 \%)\end{array}$ & $\begin{array}{l}12 \\
(75 \%)\end{array}$ & $\begin{array}{l}33 \\
(17.3 \%)\end{array}$ & $\begin{array}{l}0.72 \\
(0.019-0.272)\end{array}$ & 0.001 \\
\hline Fever & $\begin{array}{l}123 \\
(59.7 \%)\end{array}$ & $\begin{array}{l}16 \\
(100 \%)\end{array}$ & $\begin{array}{l}107 \\
(56.3 \%)\end{array}$ & $\begin{array}{l}3.68 \\
(0.001-0.0013)\end{array}$ & 0.99 \\
\hline
\end{tabular}

Out of 206 study participants, chest X-ray investigation was done for 46 patients whose AFB smear negative and still complain of sign symptoms of pulmonary tuberculosis as prescribed by health care workers, not for the purpose of the study.

About 54.3\% (25/46) had chest X-ray abnormality by health workers decision at selected public health centers. Of 25 study participants which had X-ray abnormality, 15 (60\%) were culture positive for Mycobacterium tuberculosis and 9 (40.9\%) of study participants were on the anti-TB drug even if their culture result were negative. And one study participant whose culture results positive was not treated as TB patient and treatment delayed. One previously culture result of Nontuberculosis mycobacterium was contaminated after use of Amoxicillin for 7 days. 
Table 6: $2 \times 2$ tables for comparison of culture result with health care worker decision for diagnosis of SNPT among study participants, 2017 .

\section{LJ Culture Result}

\begin{tabular}{|c|c|c|c|c|}
\hline $\begin{array}{l}\mathrm{HCW} \\
\text { decision }\end{array}$ & Positive & negative & & \multirow{4}{*}{$\begin{array}{l}\text { Sensitivity }=(A / A+C)^{*} 100=92.8 \% \\
\text { Specificity }=(D / D+B)^{*} 100=95.2 \% \text { Positive } \\
\text { Preedictive value }=(A / A+B)^{*} 100=59 \% \\
\text { Negative predictive value }=(D / D+C)^{*} 100=99 \%\end{array}$} \\
\hline SNPT & $13 \mathrm{~A}$ & $09 \mathrm{~B}$ & 22 & \\
\hline \multirow[t]{2}{*}{ Not SNPT } & $01 \mathrm{C}$ & $182 \mathrm{D}$ & 183 & \\
\hline & 14 & 191 & 205 & \\
\hline
\end{tabular}

Hypothesis testing showed no significant difference in the use of broad-spectrum antibiotics for SNPT diagnosis (Table 6).

\section{DISCUSSION}

Like other developing countries, the diagnosis of pulmonary tuberculosis in Ethiopia relies mainly on clinical screening, CXR, and sputum smear examination. While recommended by the World Health Organization (WHO) for diagnosis of AFB smear-negative TB in HIV-infected patients, a sputum culture is still not routinely available at the health center [2].

This study showed that a significant proportion of prevalence of culture-confirmed SNPT of 7.8\% (16/206) and 6.8\% (14/205) before and after taking a broad-spectrum antibiotic for 7-10 days.

Our study almost similar burden of culture-confirmed SNPTB with a study done at Cambodia a prevalence of 9.9\% [7]. And lower prevalence of smear negative culture positive PTB seen in Vietnam, which is $5.6 \%$ [8]. Higher prevalence of smear negative culture positive PTB saw in a study conducted in Ethiopia at St. Peter TB specialized Hospital, 43/247 (17.4\%) patients whose smears negative for acid-fast bacilli found to be positive for mycobacterial culture [9]. Similarly, higher prevalence of (17.5\%), (26.8\% ) and (27.8\%) smear negative culture positive results seen in Kenya, Tanzania and Peru respectively [10-12]. The variation of prevalence of SNPT may be a due difference of study population, the status of the patient and method of diagnosis.

Our study identified 1 (7.1\%) non-tuberculosis mycobacterium and 16 (92.9\%) Mycobacterium tuberculosis before taking antibiotics compared to another study done in Ethiopia by Desta $\mathrm{K}$ et al., the Mycobacterium species identified were $\mathrm{M}$. tuberculosis $(\mathrm{n}=40)(93 \%)$ and non-tuberculosis mycobacteria $(n=3) \quad(7 \%)$ [9]. Study in Vietnam found only two nontuberculosis mycobacterium cases and 11 (3.2\%) nontuberculosis in Peru [8,12]. Our study contamination rate of culture was 10 (4.85\%), which is within the acceptance range of $<5 \%$.

All smear negative culture positive PTB cases were susceptible to the first-line drug of isoniazid and rifampicin. In contrast with study done in Vietnam among 22 AFB smear-negative culture positive PTB cases, 7 (32\%) were classified as resistance to TB; of which, 5 cases were resistant to streptomycin, 1 case was resistant to streptomycin and isoniazid, and 1 MDR-TB case which was resistant to streptomycin, rifampicin, isoniazid, and Ethambutol [8].

Although our finding could not find resistance pattern of isoniazid and rifampicin it suggests other rapid and accurate diagnostic tools for detecting resistant TB cases in patients having AFB-negative sputum smears but culture positive in addition or as an alternative to line probe assay.

The common symptoms of $\mathrm{TB}$ combined with the poor specificity of X-ray screening may result in false diagnoses and people without $\mathrm{TB}$ being enrolled on $\mathrm{TB}$ treatment when it is not needed. Furthermore, a low rate of laboratory confirmation reflects under-diagnosis of true TB cases and contributes in part to the continuing global gap between notified an estimated incident TB cases: 6 million and 9.6 million in 2014, respectively [2].

In Northern Ethiopian prisons, Contact history to TB patients in prison, educational level, cough and night sweating were found to be predictors of TB positivity among smear-negative pulmonary TB cases $(\mathrm{p} \leq 0.05)(140)$. Which is in line with this study night sweat $(\mathrm{OR}(95 \% \mathrm{CI})=0.113(0.027-0.474)$ and weight loss $(\mathrm{OR}(95 \% \mathrm{CI})=0.72(0.019-0.272)$ have shown significantly associated with confirmed SNPT.

Of the 313 PTB suspects who received an antibiotic, 85\%, had a partial response. Patients with no response to the antibiotic were more likely to be culture-confirmed (OR: 3.8, 95\% CI: $1.3-10.8$, $\mathrm{p}=0.014)$ than patients with a complete or partial response. However, 9\% of the culture-confirmed PTB patients who received an antibiotic had a complete response [10].

Study in Tanzania showed that patients whose sputum smears were AFB negative were evaluated using the above algorithm by the treating doctors at the clinics or hospital. A presumptive diagnosis of AFB sputum smear-negative PTB was made in $41.8 \%$ (173) of all study subjects, and patients started on anti-TB treatment as recommended by the Tanzania NTLP. The remaining $58.2 \%$ (240) patients were assumed to have other forms of respiratory diseases and were treated accordingly [11].

Our study showed 22 (10.7\%) study participants were presumed to have active TB and started anti TB drug by health care 
workers but 9/22 (40.9\%) were bacteriological culture negative and $0.48 \%(1 / 205)$ not treated as active SNPTB and treatment delayed. Similar Study in Tanzania shows Less than half (38.1\%) of those who were presumed to have active TB and started on Anti TB actually had TB by sputum culture results. More than $60 \%$ of these patients received 8 -months of treatment despite having negative culture results. This is similar to what has been reported before in Malawi where it was reported that $40 \%$ of smear-negative had TB confirmed microbiologically $[10,13]$.

The current diagnostic algorithm leading to the establishment of the diagnosis of AFB smear-negative PTB is inefficient; it overdiagnoses PTB and misses people with active disease. Instituting a more sensitive diagnostic tool will prevent the unnecessary cost of treating individuals who do not have $\mathrm{TB}$ and at the same time, it will prevent the further spread of TB. This emphasizes the need for culture and the need for further research in order to identify a better diagnostic tool for diagnosis of AFB negative PTB.

\section{Strength and limitation of the study}

Culture done two times before and after antibiotic use can be seen as a strength. Health care workers decision on the diagnosis of SNPT compared to culture results of patients were made after the study participant followed up to finishing administration of antibiotic or CXR result, further follow up may give another scenario. The Lowenstein-Jensen solid culture media used in our study may miss some PTB cases, Liquid-based culture, MGIT should be deployed as a more rapid and accurate alternative for Löwenstein-Jensen technique. Health care workers decision could vary and affect the results. The cross-sectional study may not fully indicate the effect of the antibiotic, so cohort study may give an accurate effect of antibiotics on patients culture result. HIV statuses of the study participant were not done due to the stock out of rapid HIV diagnosis kit during the study period. Some Study participant did not come after taking broadspectrum antibiotics, so that could not achieve the desired sample size.

\section{CONCLUSION}

The study revealed that the prevalence of culture-confirmed SNPT before taking antibiotics was $7.2 \%$ and the remaining $92.8 \%$ of cases of SNPT suspects etiologically not explained by culture. The prevalence of SNPT among sputum smear-negative patients after taking broad-spectrum antibiotics was $6.8 \%$. The study does not show a significant effect of antibiotics on the diagnosis of SNPT cases among the study participants. Overdiagnosis as SNPT seen by algorithm followed by health care workers decision compared to LJ culture results.

Further study on cohort and with large sample size and in the wide area should be done to show the effect of administration of antibiotics to patients for diagnosis of SNPT. There should be an improvement in the practice of Sputum Sample collection, processing, and microscopy reading. Use of other existing better diagnosis in addition to $\mathrm{ZN}$ staining technique of $\mathrm{AFB}$ microscopy like Fluorescence and LED technology, use of culture, immunological methods (TST, IGRA), molecular technique (X-pert MTB/RIF) if visible for diagnosis of SNPT.

\section{REFERENCES}

1. World Health Organization. Global tuberculosis control report. Geneva. 2011.

2. Siddiqi K, Lambert ML, Walley J. Clinical diagnosis of smearnegative pulmonary tuberculosis in low-income countries: the current evidence. Lancet Infect Dis. 2003;3:288-296.

3. Steingart KR, Vivienne N, Henry M, Hopewell PC, Ramsay A, Cunningham J, et al. Sputum processing methods to improve the sensitivity of smear microscopy for tuberculosis: a systematic review. Lancet Infect Dis. 2006;6:664-674.

4. World Health Organization. Global tuberculosis control report. Geneva. 2015.

5. Mengiste M, Tesfay W, Madeley JR. The quality of tuberculosis diagnosis in districts of Tigray region of northern Ethiopia. J Health Dev. 2006;19:13-20.

6. Strong BE, Kubica GP. Isolation and identification of Mycobacterium tuberculosis: a guide for the level II laboratory. Centers for disease control (CDC) United States 143. 1981.

7. Tamhane A, Cheng P, Dobbs T, Mak S, Sar B. Predictors of smearnegative pulmonary tuberculosis in HIV-infected patients, Battambang, Cambodia. Int $\mathrm{J}$ Tuberculosis Lung Dis. 2009; 13:347-354.

8. Nguyen D, Nguyen H, Beasley R, Charles E, Edward A, Hwang LY. Performance of clinical algorithms for smear-negative pulmonary tuberculosis in HIV-infected persons in Ho Chi Minh City, Vietnam. Tuberculosis Res Treat. 2012.

9. Desta K, Asrat D, Lemma E, Gebeyehu M, Feleke B. Prevalence of smear-negative pulmonary tuberculosis among patients visiting St. Peter's Tuberculosis Specialized Hospital, Addis Ababa, Ethiopia. Ethiop Med J. 2009;47:17-24.

10. Helena H, Francis V, Eric O, Mathieu B, Elisa A, Sitienei J, et al. Performance of the 2007 WHO algorithm to diagnose smearnegative pulmonary tuberculosis in a HIV prevalent Setting. PLoS One. 2012;7:e51336.

11. Nyagosya R, Andersen A, Vanleth F, Pascal M, Mugomela A, Friis H. Risk factor for smear negative and culture positive results among pulmonary tuberculosis patients in Mwanza, Tanzania. Open Tropical Med J. 2008;1:68-73.

12. Soto A, Soleri L, Draz J, Mantilla A, Matthys F, Van Der Stuyft P. Validation of a clinical radiographic score to assess the probability of pulmonary tuberculosis in suspected patients with negative sputum smears. PLoS One. 2011;6:e18486.

13. Mesfin MM, Tasew WT, Richardsfini JM. The quality of tuberculosis diagnosis in districts of Tigray region of northern Ethiopia. Ethiop J Health Dev. 2003;19:13-20. 